\title{
An empirical study of healthcare providers and patients' perceptions of electronic health records
}

\author{
Giovanni Comandé, Luca Nocco*, Violette Peigné \\ Lider-Lab, Scuola Superiore Sant'Anna di Pisa, Studi Universitari e di Perfezionamento, Piazza Martiri della Libertà, 33, I-56127 Pisa, Italy
}

\section{A R T I C L E I N F O}

\section{Article history:}

Received 4 February 2013

Accepted 24 January 2014

\section{Keywords:}

Information and communication technology

Healthcare

Electronic health records

Patient

Healthcare provider

Statistical study

\begin{abstract}
A B S T R A C T
This paper is the result of a legal and policy analysis of a statistical poll focused on the area of Livorno conducted by the LIDER-Lab of the Scuola Superiore Sant'Anna (Pisa, Italy). Information were gathered by submitting questionnaires to both healthcare providers and their patients. The scope was to evaluate the interest engendered by the application of technology on health data processing along with the needs, expectations and concerns of patients and healthcare providers. The paper leads to the main policy proposals of increasing financial investments in e-health (or at least preserve this area from the generalized budget constraints public health is suffering nowadays) and introducing incentives to use computers for general practitioners.
\end{abstract}

(c) 2014 Elsevier Ltd. All rights reserved.

\section{Introduction}

Electronic Health Record (EHR) systems are one of the most important Information and Communication Technology (ICT) based solutions for the healthcare sector. They represent a new form of communication and management of patients' health data. The so-called EU Article 29 Working Group defines an EHR as follows: "a comprehensive medical record or similar documentation of the past and present physical and mental state of health of an individual in electronic form and providing for ready availability of these data for medical treatment and other closely related purposes" [2]. ICT allows for the accumulation, in a single electronic document, of all the health data of a person in order to improve their access and use by authorised individuals and third parties when required. Such an improvement is better understood considering how EHRs differ from traditional documentation on medical treatment and health data. Traditional medical records, created and stored by health care providers or organisations, are restricted to certain types of data in function of medical specializations and the actual service provided. Furthermore, they do not allow a global vision of the patient's health conditions. An EHR, instead, aims to gather health data, potentially generated by different sources at different times, and to share those data with relevant healthcare actors.
EHR is also different from Personal Health Records (PHR) $[15,23,25]$, another application of ICT in the field of health data management. The distinction is stressed by the term "personal". While an EHR is maintained by health professionals and official agencies, PHR is a collection of health-related information documented and maintained by the individual to whom they pertain (data subject), using the service offered by a provider. The most famous PHR is Microsoft HealthVault, ${ }^{1}$ available in the US and in the UK, which aims to empower the data subjects to better manage their medical data. The patient can manage PHR with no third party intervention on the records. She directly enters on the PHR health data such as blood type, blood pressure, vaccinations, drugs used and previous diseases. The accuracy of such medical data is her responsibility. The patient can decide to never share her PHR, or to share it with her relatives and/or her physicians via online health services.

It is important to notice that in Italy, PHR is not diffused. However, Italian public authorities are developing systems of EHR in order to take advantages of interoperable databases of medical data. The main benefit anticipated by the digitalisation of medical records is better coordination of treatments. EHRs enhance the quality of care allowing speedy access to the comprehensive medical history of patients. In addition, health data ubiquity can reduce potential duplication of medical tests and errors caused by poor circulation of information $[3,21]$. In addition to this, public authorities expect that EHRs will help to provide faster and more efficient health services to citizens and to

\footnotetext{
${ }^{1}$ It is significant to note that Google decided to discontinue its PHR, Google Health, for commercial reasons from 1st January of 2012 (see www.google.com/ health).
}

\footnotetext{
* Corresponding author. Tel.: + 39 050883547; fax: + 39050883530

E-mail address: lucanocco@sssup.it (L. Nocco).

URL: http://www.lider-lab.org (L. Nocco).
} 
reduce the costs of healthcare systems [12,22,27]. Finally, greater control of health data can enhance a patient's awareness about her own state of health $[1,26]$.

The expectation since the late 1990s has been that EHRs ought be integrated, on the basis of the multiple advantages they offer, into most healthcare systems $[5,11,14]$. The European Union, in proposing a strategic plan concerning ICT adoption in the healthcare sector, ${ }^{2}$ has begun promoting the creation of EHR systems in all Member States. The first step in this process was the implementation of Directive 2011/24/EU on the application of patients' rights in cross-border healthcare. It purported that personal health data should be able to flow from one Member State to another so as to ensure continuity of care. ${ }^{3}$ In the article dedicated to eHealth, the EU articulates its support for coordination between Member States in creating an "E-Health network". ${ }^{4}$

Against this backdrop, the present study has the more limited scope of illustrating the impact of the application of digital technologies to the healthcare sector exploring two specific issues. On the one hand and as a preliminary step, it investigates the actual level of knowledge of patients and general practitioners on the topic of computer applications to the processing of health data. On the other hand, it focuses on the needs, expectations and concerns of patients and professionals, at least as these two groups perceive them. Although its scope is more limited the study concludes calling for further analysis on the impact PHRs and EHRs can have on the standard of care in medical liability and for the providers of technologies and services related to the use of EHR (e.g. Internet service providers, software engeneers, etc.).

In particular, bearing the international background of e-Health in mind, our focus has been to investigate the actual perception of EHRs by professionals and patients in a small, defined geographical area. For this reason, we chose Livorno, a medium sized Tuscan city of approx. 160,000 inhabitants, as a suitable area for the dissemination of a questionnaire specifically drafted for the poll. These questionnaires were submitted to both general practitioners and their patients. The general scope of this empirical experiment was to evaluate the awareness of EHRs and determine the demands, expectations and fears of both categories of health actors. Although the questionnaire and the interview questions were not drawn up with a specific catchment area in mind, we cannot automatically claim that analogous results would be found in other local or national contexts because several social and demographic variables could influence some results. Yet, if we project the results found at the national level or at comparable catchment area, we consider that our research stresses interesting issues for a larger debate among health actors and policymakers concerning the generalisation of technological application in the healthcare sector. Analytical results will be further discussed in Sections 4-8 after having briefly described the Italian normative background in Section 2 and the empirical study methodology in Section 3. To avoid excessive length of the article, tables refer to what we think are the most interesting data resulting from the questionnaire. Finally, Section 9 focuses on policy and legal analysis stressing the need for further research on the impact HER can have on professional and ISP providers liability.

\section{The legal background to electronic health records in Italy}

In Italy, the State, Regions and local authorities share competence on healthcare. According to article 117 of the Italian Constitution, the

\footnotetext{
${ }^{2}$ See Decision no. 1786/2002/EC; Communication $\operatorname{COM}(2004) 301$; Communication COM (2004) 356; Recommendation no. 2008/594/EC; Communication $\operatorname{COM}(2008) 689$.

${ }^{3}$ See articles 4 (f) and 5 (d) Directive 2011/24/EU.

${ }^{4}$ See article 14 Directive 2011/24/EU.
}

introduction of EHRs is a competence belonging to the Regions. Nevertheless, at the national level, the Innovation and Technology Department and the Health Department created a special committee comprised of representatives from all Regions to investigate the prospects of harmonized digitalisation of the health sector.

Recently, a definition of "Fascicolo Sanitario Elettronico" (EHR) was introduced by Article 12 of the Decree 18 October 2012 no. 179 , establishing the scope, the controllers and the recipients of health data processing. ${ }^{5}$

Anticipating this legislative definition, the Italian Data Protection Authority in 2009 established guidelines on EHRs aimed at protecting citizens' privacy [9]. These privacy guidelines were recently further bolstered by national Guidelines on EHRs by the Health Department.

At the moment, the Italian infrastructure of EHRs is based on the federation of the regional architectures established in Italian regions which is called to guarantee the localisation and management of patient health data. Communication among the regional systems is then permitted via the "sistema pubblico di connettività", a public infrastructure. While some regional systems are quite well developed (for example, Lombardy, ${ }^{6}$ Emilia-Romagna ${ }^{7}$ and Tuscany $^{8}$ ) the interconnectivity of EHRs is not yet a reality at national level, revealing that the Italian system is running behind in dealing with these issues and signalling even lack of awareness of potentialities and concerns in electronic health records as our research illustrates.

\section{Research methodology}

Indeed, the hypothesis sustaining our research is that one of the most important difficulties in the implementation of EHRs in Italy is the capability of patients and physicians to adopt health data innovation.

In order to understand the perception of health actors when faced with EHRs, the Lider-Lab of the Scuola Superiore Sant'Anna, Pisa (www.lider-lab.org), with the help of the Italian Medical Association, of the General Practitioners Association of Livorno and the financial support of the "Cassa di Risparmi di Livorno Foundation", redacted and submitted two different questionnaires: one for patients and another for general practitioners. We decided to execute the study in Tuscany, a Region sufficiently advanced in the area.

A casual sample of 56 general practitioners from Livorno was selected by stratifying, in a proportional way, and considering the geographic position of the said practitioners (i.e. city centre/suburbs). The average age of the interviewees was 55 , the majority of whom were male ( $83.9 \%$ male and only $16.1 \%$ female) a differentiation which was in accordance with the GP catchment area. The sample declared an average of 28 years of professional practice. This data is important because most interviewed physicians started to practice in the early 1980s and thus acquired significant experience in "traditional" ways of prescription and maintaining patients' health records.

From this larger set of physicians, we extracted 45 general practitioners and chose our patient catchment from their patient files. We chose to interview patients in GP waiting rooms in order to reproduce the number and the frequency of consultations. The interviews were conducted during week days (from Monday to Friday) both in the morning and in the afternoon so as to capture

\footnotetext{
5 "The set of health and social health data and digital documents generated by present and past clinical events related to a patient".

${ }^{6}$ See: SISS: www.siss.regione.lombardia.it.

7 See: SOLE, Sanità On Line: www.progetto-sole.it.

${ }^{8}$ See: www.regione.toscana.it/cartasanitaria.
} 
all typologies of patients as it was presumed that younger and working patients attend general practitioners at different times from elderly and retired ones. A total of 268 patients were interviewed.

The average age of patients was 55 and the majority were female (61.2\%), with an absolute majority of married people $(67.4 \%)$ vs. non-married (19.3\%), separated (6.8\%) and widowed (6.4\%). In relation to education, $39.6 \%$ of the interviewees held high school certificates, $15.8 \%$ held university degrees, $13.1 \%$ a primary school certificate and $31.5 \%$ held a junior high school certificate. On a final note, the patients interviewed declared that they attended general practitioners on average 8.62 times a month. This value is on average rather high.

Having described our catchments, we must now turn to describe the answers to four of the several questions we asked in the interviews to both sets of interviewed individuals (physicians and patients). We selected for this paper these data since they illustrate actual awareness of the role HRS can play in healthcare and their potential impact on liability issues and physicians' behaviour in minimizing the risk of error. The data reported here illustrate the problematic nature of the shift from paper-based health data records to electronic ones urging for further research.

\section{Who has ever heard about EHRs?}

One of the first questions asked to general practitioners and patients regarded their awareness of EHR. ${ }^{10}$

Having said that, in 2010, a high percentage of general practitioners knew what an EHR was (80\%). This encouraging result can perhaps be linked with the computer-savvy nature of healthcare providers in Livorno. Indeed, $86 \%$ of the GPs had been regularly using computers in their practice for more than 7 years at the time of interview; mostly for its usefulness (58.1\%) and only a minority according to contractual and organisational obligations (32.6\%) or for data security (9.3\%).

It is interesting to note that the percentage of general practitioners in Livorno using computers exactly matches the results of the European Commission survey regarding the use of data processing services in health care. In this EU poll, 86\% of Italian general practitioners operated computers, on par with the European average (87\%).

The pervasive use of computers and the growing adoption of e-Health tools and services among the interviewed GPs is an important data. However, it does not undermine the need to improve and generalise computer use and ability by GPs. This need was supported by the same set of general practitioners, since $76 \%$ of them stressed the request for more education and training in this field.

Among patients, the knowledge of EHRs was less pervasive since only $59.7 \%$ had ever heard about it. Nevertheless, this data is not totally negative considering the novelty of the technology and that most governmental propaganda initiatives were subsequent to the survey.

\footnotetext{
${ }^{9}$ Considering the number of the sample, the statistical analysis uses the traditional procedure of asymptotic inference (with the opportune correction for the sample). For the analysis of the data we used the $R$ statistical software (www.r-project.org).

${ }^{10}$ It is appropriate to specify that, at the interview time, an information campaign was in progress (see http://www.regione.toscana.it/cartasanitaria/fascicolo/cose/index. html) but the Tuscan Regional System of EHR was not totally implemented. We consider that this detail could influence the result of our research and the result could actually be different in the future with the development of EHRs. It could be interesting to repeat the research in the coming years in order to evaluate any changes in perception.
}

At large, the results point out that a large information campaign is fundamental to enable a wider EHR use by all patients. In addition, the survey suggests that policymakers should act in an attentive manner when it comes to aged people who perhaps do not have ready access to computers nor sufficient ability to overcome ICT illiteracy. This is a matter of concern because elderly individuals generally require more healthcare in comparison to other groups but are the ones who suffer a more significant "digital divide" when juxtaposed with the younger generation [20].

\section{Electronic health records: an improved control of health data}

The survey reveals that proper information is a key factor in fostering acceptance of EHRs. Indeed, after a brief description of what EHRs are, many of the interviewed patients recognised their usefulness (91.5\%). This result may be related to the answers given when questions of errors in recording health data were raised. In fact, $64 \%$ of general practitioners admitted to having made mistakes on average 27 times over the last 5 years when recording health data in hard-copy form (Fig. 1).

In $35.5 \%$ of these cases, the error related to patients' personal data and, in the same proportion, formal data in the prescription. Inaccuracy in the content of the prescription was discovered in $29 \%$ of the cases (Fig. 2).

In most of these cases, pharmacists discovered the mistake (58,1\%). However, in some cases the patient (12.9\%), the healthcare organisation $(19.4 \%)$ or the practitioner him/herself $(9.7 \%)$ realized an error was made (Fig. 3).

$72 \%$ of physicians purported not to have had any complaints from patients, pharmacists or other health care professionals regarding the comprehension of their handwritten prescription. However, a vast majority of GPs (89.6\%) did not consider erroneous prescriptions or poor legibility as an incorrect health care diagnosis or therapy. Possibly such an high perception of erroneous prescriptions or poor legibility is related to the fact that in most cases mistakes in data did not generate adverse effects since the

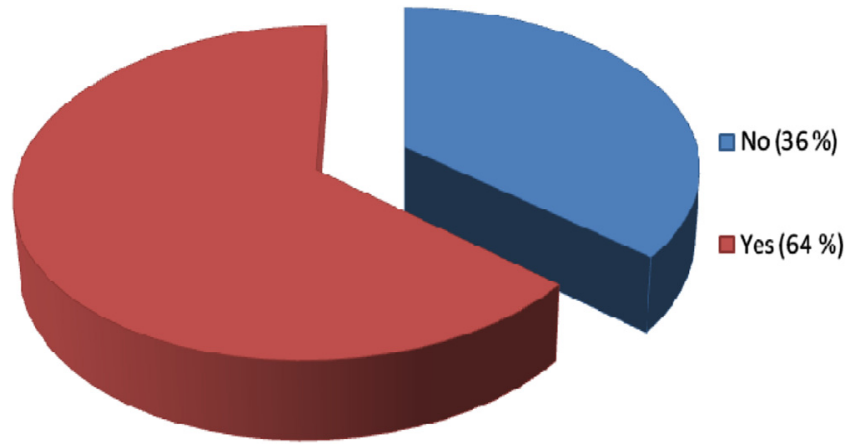

Fig. 1. Did you made any mistake in the last five years in recording health data in hard-copy form?

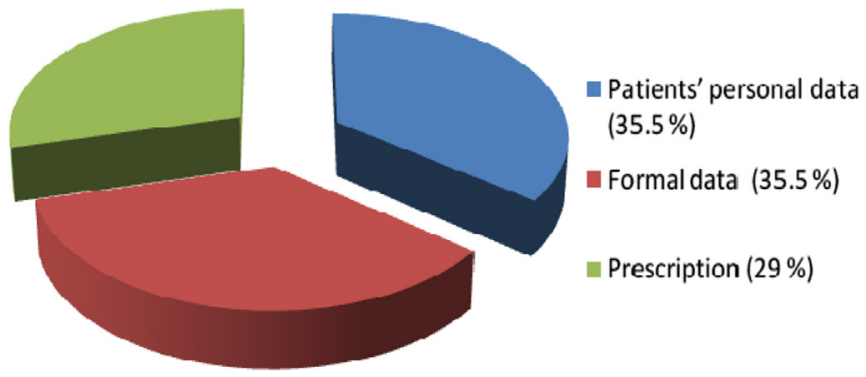

Fig. 2. The error was related to... 


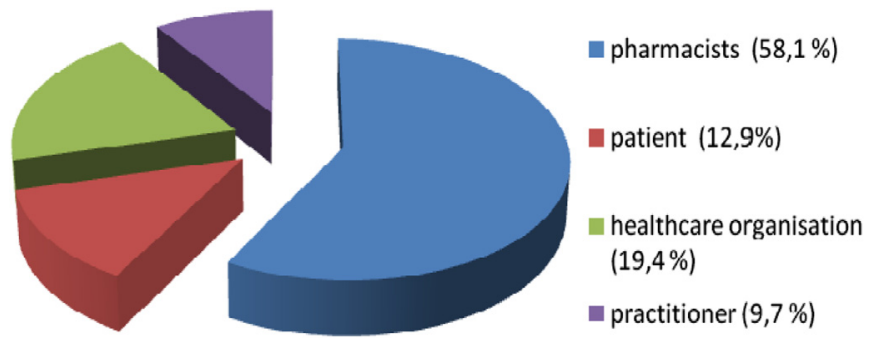

Fig. 3. Who discovered the error?

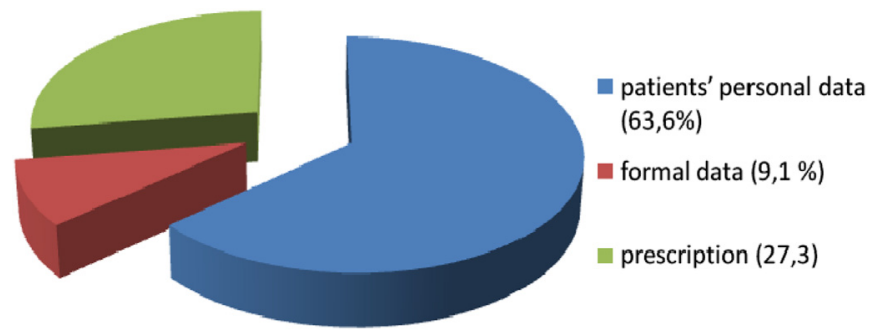

Fig. 4. In case of computerised prescriptions, the mistakes were related to...

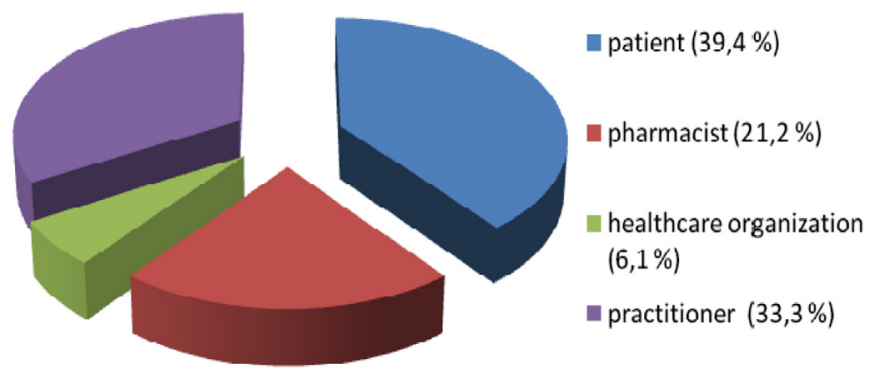

Fig. 5. In case of computerised prescriptions, who discovered the error?

errors were detected by a third person (as noted above, a pharmacist, a patient or another healthcare provider).

Nevertheless, most general practitioners $(85.1 \%)$ were of the opinion that the use of computers reduces the incidence of errors in that it simplifies the reading and filing of health records and prescriptions. There was a substantial consciousness of the usefulness of computers to minimise risks related to health data.

However, the persistent high percentage (14.9\%) of physicians who believe that the use of paper (and not of computers) reduces the incidence of errors is a significant data. It means that a large segment of professionals has yet to receive appropriate computer education. Law makers and healthcare managers should therefore invest more on this aspect even in a time of financial budget cuts.

Of course, recording health data using a computer is not totally safe either. In fact, $75 \%$ of the interviewees admitted having made mistakes while recording data in electronic form.

In order to compare handwritten and computer recording, we asked the same questions regarding the typology of errors and their eventual identification with reference to computerised prescriptions.

Accordingly, and with reference to the 5 years prior to this research, physicians estimated that on average, $25.8 \%$ of their interactions with patients were erroneously recorded in some way. The mistakes reported concerned inaccurate recording of patients' data (63.6\%), formalities (9.1\%) or incorrect prescriptions (27.3\%). (Fig. 4).

This result was only slightly lower than that related to handwritten recording (an average of 27 cases in the last 5 years). Thus

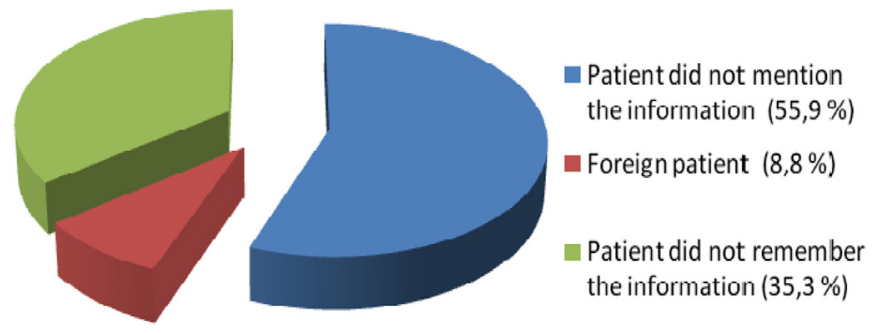

Fig. 6. In case you have ever needed to know about any medical data of your patient and were unable to acquire the information, that was due to:

it could not justify the belief that technology is sufficient to avoid mistakes. Comparing errors in manual and computer recording, we uncovered substantial homogeneity: $29 \%$ for the manual and $27.3 \%$ for the computerised prescription.

As one would expect, formal mistakes dropped from $35.5 \%$ when considered handwritten prescriptions to $9.1 \%$ when juxtaposed with its computerised counterpart. While this is to be welcomed, the incorrect recording of patients' data was significantly more common in the computerised scenario (63.6\%) when compared with manual documenting (35.5\%). This result may be read in different ways. It could simply be the result of one error in the initial recording of personal data in the patient's file, an error that automatically echoes in all subsequent prescriptions. Another explanation might be, for instance, the very fact that a high percentage of GPs have been somehow "forced" to the use of IT (86\%) means they are perhaps more "relaxed" or absent-minded when it comes to electronic recordings, perhaps even overtrusting the use of technology itself.

These considerations must be borne in mind when it comes to EHR education and training programs for which, as we mentioned earlier, most interviewed general practitioners asked for.

Furthermore, the fact that errors have been detected in the use of EHR suggests that the use of EHR should be first introduced experimentally allowing professionals to "familiarize" with these "new" instruments and only after transformed in default working tools.

In comparing handwritten and computerised mistakes, it is noteworthy that there are significant changes concerning who eventually noticed the errors: patients (39.4\%) followed by GPs (33.3\% of cases), pharmacists (21.2\%) and healthcare organizations (6.1\%) (Fig. 5). It is interesting to highlight that in relation to computer compilation, the discovery of mistakes by patients (39.4\% vs. $12.9 \%$ ) and by general practitioners (33.3\% vs. $9.7 \%$ ) is significantly higher than for handwritten mistakes.

This result reveals that computerized compilation facilitates verification of health data and the detection of mistakes. This assumption seems reasonable as it is easier to read information recorded on a computer as opposed to handwritten information, particularly for patients.

We also asked general practitioners whether they would continue to use the same modality they are accustomed to or would change their ways on discovery of errors in relation to either mode of prescribing i.e. computerised or manual. The answers we received were quite varied. General practitioners familiar with handwritten compilation answered that they would adopt the technologically advanced mode i.e. computerised prescriptions (57.7\%). On the other hand, if the error were the result of using the computerised mode of prescribing, only $20.9 \%$ would return to paper format. This result reveals that physicians are conscious that their switch to advanced technology has no way back. It illustrates also they are aware of the benefits, in the interests of patients, that enhanced third party control over health data can bring. Such an advantage cannot be undermined. 
However, our survey suggests we must be mindful that computer use by itself is not capable of preventing all possible errors.

Despite the actual relatively low percentage of consequences recorded in terms of mistaken healthcare diagnosis or therapy caused by the incorrect recording of health data (10.4\%), it has to be considered that even only one adverse event can be very damaging for the patient. Thus, it is important to improve computer education and training of general practitioners so as to minimise the risks of error.

Public interest in protecting health and reducing the cost of medical errors could lead to the fostering of EHRs. However, such a move would require that every general practitioner has her own computer well connected to the Internet and equipped with adequate software. Such a computerisation presents costs in terms both of education and hardware/software acquisition that requires the design of a varied set of incentives. Public authority can certainly choose to force healthcare providers and organisations to modernise their information systems. Yet, the majority of the interviewees (63.3\%) were against compulsory adoption of EHRs without proper incentives. We are also of the opinion that a mere policy of compulsory adoption of EHR would not be the most effective in improving the quality of health records. Proper incentives must be devised; for instance, it may be more appropriate to introduce economic incentives along with education and training programs at regional or local levels leading to compulsory adoption of EHRs. As a consequence our research suggests to policy makers and healthcare managers to invest in e-health, preserving it from financial cuts, taking into account the cost savings and safety improvements that it can produce in the long term.

\section{EHRs as an instrument to improve health communication}

As mentioned before, the need to share information on health is also related to the evolving conditions of healthcare services that are ever increasingly characterized by multidisciplinary approaches and technologies. The increasing number of actors and the increasing specialisation of treatments, together with expanding inter-regional and transnational outbound patient mobility [4], amplify the risk of health data dispersion and the need for ICT tools. Moreover, today, physicians rarely have access to the entirety of the data when it comes to a patient's health condition. The absence of a full medical history may lead both to repeat diagnostic tests and to exposing patients to unnecessary or even dangerous treatments with the aggravating result of needlessly burdening the healthcare finances.

EHRs can play an important role in tackling these risks. To test the understanding of these potentialities, we asked medical interviewees whether they have ever needed to know about any possible allergies, intolerances, active illnesses, current treatments, case-histories, test results, etc. of a patient, or any previous prescriptions by the same physician, and if, where necessary, they were unable to acquire the named information.

The answer from $72 \%$ of interviewed GP was positive. In the mentioned scenarios, they blamed the patient for not mentioning the information (55.9\% of cases), not remembering (35.3\%) or for their inability to speak Italian (8.8\%) (Fig. 6).

In a similar way, physicians were asked whether they had ever prescribed the same diagnostic tests on more than one occasion because they could not remember or the patient had not reminded them that that same test had already been prescribed to them recently. The answer was positive in $53.1 \%$ of cases (Fig. 7 ).

Patients' answers to similar questions substantially confirmed those data and the general existence of concerning issues. Indeed, a small but significant percentage of interviewed patients declared to have repeated - by mistake - diagnostic tests prescribed by a physician (Fig. 8).
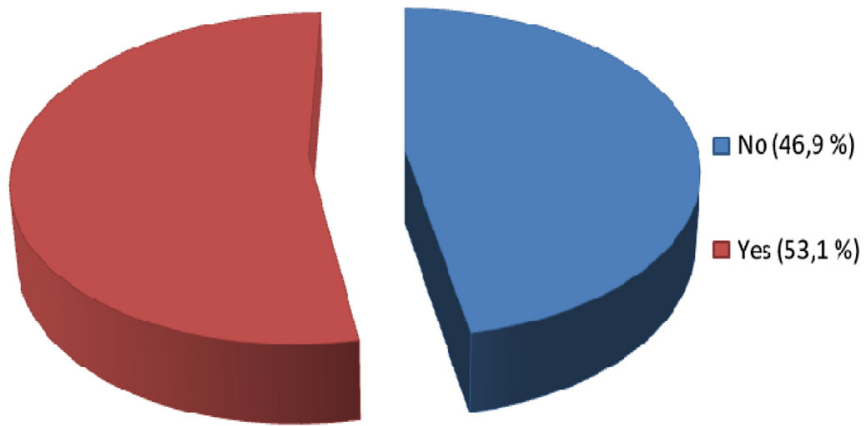

Fig. 7. Have you ever prescribed the same diagnostic tests on more than one occasion because you could not remember that it had already been prescribed recently?

The potential to make these mistakes may be easily considered, among other things, as one of the reasons for patients' desire for the implementation of a more computerised provision of health services. In their eyes, it would go a long way to reducing the risk of duplications and/or therapeutic and diagnostic errors.

From gathered data, is it easy to surmise that traditional methods of keeping records (i.e. in handwritten hard-copy) are less effective and efficient in ensuring adequate healthcare services since the traditional methods relies heavily on the recollections of both patient and doctors concerning an individual case history. The need for change towards the use of ICT is further supported when we consider the impossibility to access hardcopy records, for instance, during home visits or at a healthcare facility different than the one keeping it. The usefulness of EHR and more generally of ICT is further emphasized when we consider the high number of patients treated by each physician in Italy (up to 1400 per GP) or when we consider physicians who are faced with unfamiliar patients (for instance, patients on holiday) or physicians substituting colleagues to cover for their holidays or illness. ${ }^{11}$

EHRs may be an effective answer to all these concerns including when patients and/or doctors fail to remember their own (or their patient's) case history. Moreover, EHRs have the potential to easily overcome language gaps. Of course this latter consideration would depend on the structures introduced to coordinate various (and even non-national) systems which would have to support the readability of foreign EHRs. The development of ICT bearing cross-border readability in mind, alongside appropriate EU regulations in the field, are important factors to consider in achieving a sufficient level of EU coordination. And indeed, this is the objective of the "E-Health network"12 created by Directive 2011/24/EU on the application of patients' rights in cross-border healthcare.

These background information help to explain practitioners' answer to whether they thought that if access to a patient's EHR were available they could in fact have avoided prescribing wrong tests or repeat tests. Only $10.3 \%$ of the interviewees gave a negative answer to this question.

Nevertheless, against this backdrop of data, most doctors (63.3\%) do not want EHRs to be compulsory. This result, coupled with the other data illustrated above, leads us to the conclusion that appreciation for and interest in EHRs is overridden by physicians' concerns that it might compromise their own discretionary power. Accordingly, only $52 \%$ of interviewees believe investment in EHR by the local health

\footnotetext{
${ }^{11}$ Risk which will be probably even more concrete after the entry into force of the Article 1 of Law 158/2012 providing that family practitioners will be gradually replaced by clinics open 24/7. Doctors will alternate in such structures inevitably leading to reduced awareness about the specifics of the cases.

12 See article 14 Directive 2011/24/EU.
} 


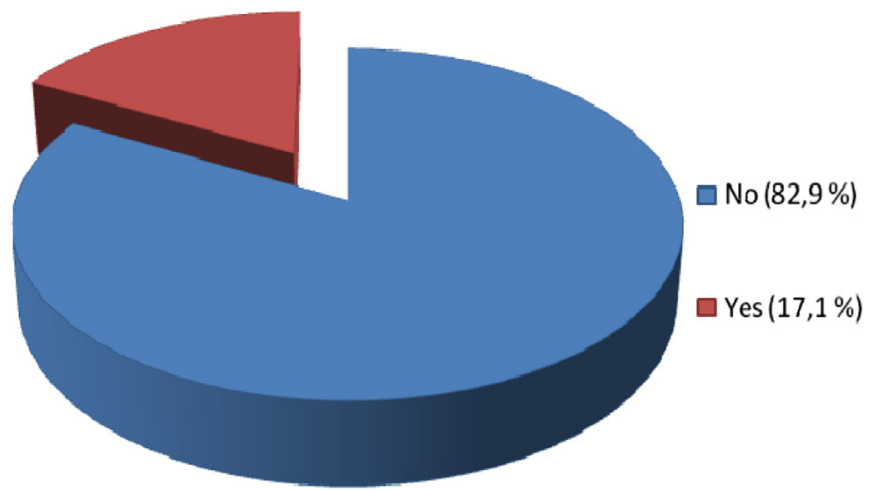

Fig. 8. Have you ever repeated -by mistake- diagnostic tests prescribed by a physician?

authority to be a priority. Indeed, any change would necessarily occur in a context according to which the healthcare system is suffering from pressing budget constraints. For this reason, physicians fear that the cost of such new instruments may rest economically on them. Of course these concerns could be overcome if the shift to computerised healthcare by public authorities would take into account the subsequent savings in drug and diagnostic treatment prescriptions to be obtained from a generalised computer application. As argued above, our research suggests financial investments in e-health should be excluded from the generalized cuts in the public health sector. To the contrary it could even be financially sound to devise economic incentives for general practitioners to use computerized systems.

\section{EHR and patient participation}

If Personal Health Records seem to be the main tool of patient empowerment, EHRs are also thought to enhance patient participation. Just like PHR, data is recorded and stored in electronic form rendering the patient's file readily accessible thanks to the Internet. With better information it is expected that patients will be more inclined to take an interest in their own health ${ }^{13}$ and play an active role in their health protection [8].

This novelty is reflected in our survey by the sharp difference between the low percentage of patients requesting access to their paper health records kept by physicians (17.5\%) and the much more significant number $(66.8 \%)$ of patients interested in gaining direct access to their EHRs.

Indeed, access to EHR does not necessarily require the intervention of a physician or of other third parties. ${ }^{14}$ This simple fact may stimulate patients to effectively enjoy their right to access and thus gain more awareness of their health status and health related behaviours. Moreover, potential direct access clearly promises significant cost savings for both the interested party and for the healthcare system. Furthermore, according to the Italian national guidelines, individuals would also be entitled to choose the recipients of their EHR since consent of the data subject (i.e. the person to whom the data refer) is a necessary condition for access to the EHR ${ }^{15}$ except in emergency cases or cases concerning the incapacity to give consent. ${ }^{16}$

\footnotetext{
${ }^{13}$ A person or her representative accesses the EHR via a computer (see Ministero della salute, Linee guida nazionali, p. 21) by using an internet porta created by each region, such as that in Tuscany (https://fset.e.toscana.it/fset).

${ }^{14}$ See Ministero della salute, Linee guida nazionali, p. 21.

15 See Ministero della salute, Linee guida nazionali, p. 19.

16 The Data Protection Authority observes that in an emergency, when a person cannot consent, the data necessary to protect her life may be consulted by all health professionals treating her. See Linee guida in tema di Fascicolo sanitario, p. 6.
}

This normative background is relevant for our analysis since under general privacy law rules data subjects are entitled to know the recipients of their health data, ${ }^{17}$ even if they cannot designate them because healthcare data access management is the responsibility of individual professionals and/or of healthcare providers holding the data.

Bearing this legal background in mind, the answers we received in relation to higher protection of the self-determination that EHRs might guarantee reveal partially contradictory data. $69.5 \%$ of patients was of the opinion that all health professionals who treat a patient must have access to their EHR. Furthermore, $76.7 \%$ of them thought that physicians should be able to access all data concerning the patients' health conditions.

However, it is also possible to assume that since individuals create their EHR to obtain better treatments, they will allow data accessibility to each physician.

Yet, these data may be interpreted in various ways. It is true that, according to answers given, patients rate data protection quite low. However, at the same time, the number of individuals asking to set limits to data access is substantial, ${ }^{18}$ confirming the need for a regulated process when it comes to data access authorisation and perhaps even data masking. ${ }^{19}$ It is noteworthy to remember that while the content of PHR is totally managed by patients, information in EHR are registered by healthcare professionals in order to ensure the accuracy of such data. Accordingly, EHR may contain a significant quantity of information introduced by a large number of professionals, potentially all those the patients have encountered in their life. In order to respect the doctor-patient relationship, where the patient revealed personal information in function of different levels of trust with the professional involved in the service, public authorities foresee the power for the patient to conceal some information in her EHR with all the imaginable consequences on risk and liability management.

Our data confirm the fiduciary nature of the doctor-patient relationship, sustaining that physician access to data is not just strictly pertinent to the current treatment even though patients appear conscious of the sensitive nature of health data in general (85.5\%). This result is probably due to the conviction, on the side of patients, that a broader knowledge of health conditions may produce better outcomes.

It is worth mentioning that $54 \%$ of interviewed physicians are in favour of their accessibility to patients' EHRs, regardless of their consent, under the assumption that the pursuit of on-going treatments and on-going contractual relationships allow it. Likewise, $64 \%$ of them think it is better that, on accessing an EHR, a physicians should be allowed to view the entire content of the patient's EHR thereby excluding the possibility of masking certain information.

The comparison between the responses of physicians and patients confirms that patient confidence in physicians seems to justify a lower attention to privacy concerns. Actually, and puzzlingly, the results show that more patients than medical doctors (69.5\% of patients vs. $54 \%$ of physicians) are of the opinion that all health professionals should be able to access EHRs. The question concerning whether physicians should be able to access the full content of the EHR highlights a similar conclusion $(76.7 \%$ of patients vs. $64 \%$ of interviewed GPs).

We surmise two possible explanations of this apparent contradiction. On the one hand, we can infer that clinical experience leads healthcare professionals to believe that access to only those

\footnotetext{
${ }^{17}$ Article 13 Code of Personal Data Protection.

$1830.5 \%$ of interviewed patients think that not all professionals treating them should be able to automatically access their EHR and 23.3\% think that a professional with access to the EHR is not capable of absorbing all data.

${ }^{19}$ See Ministero della salute, Linee guida nazionali, pp. 19-20.
} 
records strictly relevant to current treatments is necessary. In other words, patients may prefer an indiscriminate medical access on the basis of a feeling of reliability on professionals' confidentiality, dictated mostly by patients' lack of medical science knowledge.

On the other hand, perhaps out of malice, we may assume that the well-known problems of potential medical liability may lead professionals to limit their access to health records as a defensive medicine attitude.

Technically speaking, medical doctors may be interested in avoiding liability related to health record processing and storage. Moreover, their answers could have been influenced by potential liability in the use of EHR $[4,13,16]$ and by its impact on the determination of civil and criminal liabilities (68.1\% physicians answered in the affirmative to the relevant question). In a sense, these background concerns might have led doctors to think it convenient "to hide" behind arguments related to innocent unawareness of the specifics of the health record.

Finally, we must remember that the specific question concerning whether patients should be free to withhold or mask part of their EHR content was answered positively only by $32.7 \%$ of GPs.

Therefore, issues surrounding whether a patient can choose "the person to whom" and "what" to reveal about certain health conditions are solved, at least according to interviewed GPs, by permitting the widest possible access to records. It is easy to conclude then that from the medical point of view, somehow an underdeveloped EHR risks to be just as ineffective than paper based data records.

\section{EHR and individual rights protection}

The application of computer systems to data processing and filing does not remove - but only transforms - the problems connected to patients' privacy protection. The EHR system is aimed at developing the circulation of health records, but it can result, at the same time, both in the loss of records and the loss of control over data $[4,10,28]$. The higher the circulation, the more harms may be caused to privacy, identity, and freedom and even to dignity $[13,17,19,25]$.

The analysis of the results of the questionnaire reveals good awareness of the sensitive nature of health records (85.5\%), which corresponds to a generalised aversion towards the possibility of subjects, different from healthcare providers, such as employers or insurers, accessing EHRs (92.7\%). Yet, 75.9\% of interviewees would not object to the use of their EHR for medical research, probably due to its social and unprofitable utility while it remains unclear in our survey whether or not the latter mentioned are the actual reasons of the answers obtained.

On the other hand, the need to avoid a discriminating use of EHRs may lead, as confirmed by the EHR Italian Data Protection Authority Guidelines, to prohibited access, irrespective of the patient's consent, for, as proposed, insurers and employers. Such a prohibition may be set against the possible usefulness of access to such market players, for example in order to avoid unnecessary medical tests prior to new employment or for issuing an insurance policy. A total ban on access might result in an excessive and unjustified limitation of personal freedom. Yet, this limitation to the principle of consent seems in line with the very role of consent according to the Italian Data Protection Code (Law no. 196/2003). ${ }^{20}$

Furthermore, precluding access to EHRs for insurers and employers is consistent with the aims of EHRs in that they,

${ }^{20}$ See for instance Article 20 , according to which consent is neither a necessary nor sufficient condition for processing sensitive data. according to the Italian Privacy Authority Guidelines, "may be connected exclusively with the finalities of the interested parties' treatment".

However, it has been suggested that individuals may ask their GP to extract ${ }^{21}$ from their EHR those medical records they want to communicate to an insurer/employer. Yet, this praxis would have the effect of permitting reverse discriminations on the assumption that undisclosed data conceal existing illnesses thereby putting undue pressure on data subjects to actually reveal all available data on health bypassing altogether the public policy concerns sustaining the ban against access for insurers and employers.

Finally, we must mention the possibility of illegal accesses to EHRs. As a matter of fact, technology applied to data security can reduce these risks but cannot avoid them entirely. Nevertheless, the protection of EHR security is a key element for any successful implementation of EHRs because it is a condition of their social reliability and acceptability [13].

On this issue, GPs were asked whether they ordinarily apply security measures to protect EHRs with $91.1 \%$ of them answering affirmatively. Note, however, that some existing security measures are imposed by law on all professional processing of sensitive data including those relating to health.

\section{Conclusions}

The journey of continuous healthcare system reform is at a turning point and is faced with contrasting elements. On the one hand, effective electronic health records management could satisfy new needs in healthcare services; on the other hand, pressure to reduce costs might undermine the very effectiveness of their functionality. We think it is possible, at least partly, to face the current and future challenges of national and transnational healthcare systems by expanding the reach of information and communication technologies.

EHR systems are fundamental elements of the modern healthcare systems, since they can assure a higher quality and security of health records in comparison to traditional modes of information collation, storage and transmission. Similarly, they can facilitate access to records while assuring high standards of protection and security for health care data and individual privacy. The social and economic advantages of a wider use of EHR systems are potentially high and our survey has shown that both professionals and patients are ready for a change. Our research has evidenced a sufficiently broad understanding of EHRs by health care providers and patients, even though it is also clear that further investment is required in supporting awareness of the pros and cons of the use of ICT in healthcare data management.

Moreover, the research has stressed, on the part of both actors, a clear awareness of the possible advantages offered by computerising health records. At the same time, the research has evidenced biases and concerns in relation to computer use by some professionals, potentially impacting on the entire process of digitalising healthcare data. Answers in the survey show that several of these concerns can be overridden through a sharing of the costs of the process which probably remains at the basis of any hostility from professionals to the envisaged technologically driven changes.

Simultaneously, the research confirmed the underlying intuition that involved actors perceive the potential risks of the novel technologies along with their potential benefits. Overall, the data reveals the risk of perceiving the technical innovation as a path to eradicate errors in medicine. This is an important point worthy of further research and analysis. Indeed the survey has made the case

\footnotetext{
${ }^{21}$ Such possibility is admitted by the Article 29 Data Protection Working Party Working Document on the processing of personal data relating to health in electronic health records (EHR), WP 131, 15 February 2007, p. 19.
} 
for further research on both case and statutory law to clarify the liability patterns that might arise in the use of EHR and PHR. In particular, further research should analyze the degree of accuracy and completeness of the data which is required to practitioners as well as to what extent professional or Internet service providers' liability may emerge in case access is not possible for technical reasons. The combination of case law and statutory provisions if coupled with the availability of technologies such as EHRs and PHRs can have an impact on the level of care imposed to practitioners. It might theoretically result in a further expansion of the phenomenon of the "litigation explosion" which characterizes professional liability in all modern post industrial societies.

\section{Conflict of interest statement}

The study sponsor did not have any involvement in the study design, in the collection, analysis and interpretation of data; in the writing of the manuscript; and in the decision to submit the manuscript for publication.

Therefore we declare we do not have any conflict of interests.

\section{Acknowledgments}

The research was funded by the "Fondazione Cassa di Risparmi di Livorno", an Italian no-profit organization which is not interested in the medical field.

\section{References}

[1] R. Agarwal , C.M. Angst, Patients Take Control: Individual Empowerment with Personal Health Records, Robert H. Smith School Research Paper \# 904622 2004

[2] Article 29 Data Protection Working Party, Working Document on the Processing of Personal Data Relating to Health in Electronic Health Records (EHR), WP no.131, 2007.

[3] P. Aspden, Patient Safety: Achieving a New Standard for Care, Committee on Data Standards for Patient Safety, Institute of Medicine of the National Academies Press, Washington, D.C., 2004.
[4] G. Comandé, Patients Medical Tourism and Electronic Health Records: Mobility Between Sector Regulations and Systemic Interactions Liability in the Third Millennium, in: A. Colombi Ciacchi, C. Godt, P. Rott, L.J. Smith (Eds.), Liber Amicorum in Honor of Gert Brüggemeier, Nomos, Baden Baden, 2009.

[5] A. Cornwall, Connecting Health: A Review of Electronic Health Record Projects in Australia, Europe and Canada, Public Interest Advocacy Centre, 2002.

[8] J.B. Fowles, et al., Patients' interest in reading their medical record relation with clinical and sociodemographic characteristics and patients' approach to health care, Arch. Intern. Med. 164 (2004) 793.

[9] Garante per la protezione dei dati personali. Provvedimento a carattere generale, Linee guida in tema di Fascicolo sanitario elettronico (Fse) e di dossier sanitario, 2009.

[10] L. Gostin, Health ineducation privacy, Cornell Law Rev. 451 (1995) 81.

[11] Harris Interactive, U.S. trails other English speaking countries in use of electronic medical records and electronic prescribing, Health Care News 1 (28) (2001) 1.

[12] R. Hillestad, et al., Can electronic medical record systems transform healthcare? An assessment of potential health benefits, saving and costs, Health Aff. 24 (2005) 5.

[13] S. Hoffman, A. Podgurski, Finding a cure: the case for regulation and oversight of electronic health record systems, Harvard J. Law Technol. 22 (1) (2008) $103-165$.

[14] I. Iakovidis, Towards personal health record: current situation, obstacles and trends in implementation of electronic healthcare records in Europe, Int. J. Med. Inform. 52 (123) (1998) 105-117.

[15] M.R. Kidd, Personal electronic health records: MySpace or HealthSpace ? BMJ 336 (2008) 1029.

[16] J.B. Korin, MS. Quattrone, Litigation in the decade of Electronic Health Records, Use of EHRs may raise new risks of malpractice liability, N. J. Law J. 188 (11) (2007) 845.

[17] J. Loughrey, The confidentiality of medical records: ineducational autonomy, patient privacy, and the law, North. Irel. Legal Q. 56 (3) (2005) 293.

[19] J. Montgomery, Personal ineducation in the National Health Service: the demise or rise of patient interests?" in: S. Lace (Ed.), The Glass Consumer: Life in a Surveillance Society, National Consumer Council, 2005, p. 187.

[20] E. Mordini, et al., Ethics, e-inclusion and ageing, Stud. Eth. Law Technol. 3 (1) (2009) (Article 5).

[21] National Committee on Vital and Health Statistics, Testimony, 1999.

[22] A. Solovy, The big payback: 2001 survey shows a healthy return on investment for into tech, Hosp. Health Networks (2001) 40.

[23] R. Steinbrook, Personally controlled online health data-the next big thing in medical care? N. Engl. J. Med. 358 (2008) 1653.

[25] N.P. Terry, L.P. Francis, Ensuring the privacy and confidentiality of electronic health records, Univ. Ill. Law Rev. (2007) 681.

[26] F. Ueckert, et al., Empowerment of patients and communication with health care professionals through an electronic health record, Int. J. Med. Inform. $70(2-3)(2003) 99$.

[27] S.J. Wang, et al., A cost-benefit analysis of electronic medical records in primary care, Am. J. Med. 114 (5) (2003) 397.

[28] V. Zambrano, Dati sanitari e tutela della sfera privata, Dir. Inform. (1999) 1. 